\title{
Pharmacological Analysis of Antigen-Induced Late Airway Response in Rats
}

\author{
Naoki Miyagawa, ${ }^{*}$ Hidenori Iwasaki, Toshinobu Kato, Masaru Tanaka, Tsutomu Shibata, and \\ Korekiyo WAKITANI \\ Central Pharmaceutical Research Institute, Japan Tobacco, Inc.; 1-1 Murasaki-cho, Takatsuki, Osaka 569-1125, Japan. \\ Received October 21, 2008; accepted December 18, 2008; published online December 19, 2008
}

The pharmacological and pathophysiological characteristics of rat antigen-induced late airway response (LAR) are not yet fully understood. In this study, the pharmacological properties of rat ovalbumin (OVA)induced LAR and effects of the clinically used anti-asthmatic drugs salbutamol ( $\beta_{2}$-agonist), ketotifen (antihistamine), pranlukast (anti-leukotriene $C_{4} / D_{4} / E_{4}$ ), and prednisolone (steroid) were examined. In addition, a comparison was made of cell infiltration in bronchoalveolar lavage fluid (BALF) between immediate airway response (IAR) and LAR, and the edematous features of lung during LAR were also examined. Although infiltration of inflammatory cells into BALF was increased in both IAR and LAR, only the increase in eosinophils at 1, 3, and $6 \mathrm{~h}$ during LAR were significantly higher than those during IAR. Although $\beta_{2}$-agonist, antihistamine, and antileukotriene $C_{4} / D_{4} / E_{4}$ exhibited no effects on rat LAR, steroid attenuated LAR and decreased eosinophil number in BALF. LAR and the percentage water content were both increased after antigen inhalation, suggesting that LAR is involved in pulmonary edema in rats. In conclusion, antigen-induced LAR was related to pulmonary edema and eosinophil infiltration rather than contraction of airway smooth muscle. This is the first comprehensive study of the profiles of rat antigen-induced LAR, and these analyses of LAR improve understanding of the diverse mechanisms underlying human asthmatic diseases.

Key words late airway response; antiasthmatic drug; eosinophil infiltration; edema

Bronchial asthma is one of the most common allergic diseases in the world, and is considered a complex inflammatory disorder of the airways with various pathophysiological features. ${ }^{1)}$ The exposure of asthmatics to specific antigens causes airway symptoms which can be generally distinguished into two predominant airway reactions, an immediate airway response (IAR) and a late airway response (LAR). ${ }^{2,3)}$

The pharmacological profiles of antigen-induced IAR and LAR have been determined using animal models, especially in the guinea pig. For example, it is known that antileukotrienes reduce LAR in guinea pig, while antihistamines do not. ${ }^{4,5}$ On the other hand, inhalation of a $\beta_{2}$-agonist like salbutamol, which directly inhibits the contraction of airway smooth muscle, diminishes IAR but not LAR. ${ }^{6}$ Further, antiIL-5 monoclonal antibody decrease infiltration of eosinophils into the tracheal wall, and also markedly suppresses the development of LAR in guinea pig. ${ }^{7)}$

In recent years, the pharmaceutical targets for new asthmatic drugs have become more immunologically diverse and specific, and detailed immunological analysis of mechanisms in animal models is required to determine with precision the efficacies of new drug candidates. However, the guinea pig model is not sufficient for this purpose, since immunological and genetic research on guinea pigs has not been performed in detail.

In this regard, we have examined the pharmacological and immunological characteristics of ovalbumin (OVA)-induced IAR and LAR in the rat. Briefly, rat IAR exhibits diverse immunological characteristics, i.e. a quick phase $(3-6 \mathrm{~min}$ after challenge) caused by the exocytosis of mediators in CTMCs and an early phase (6-30 min after challenge) mediated by leukotriene (LT) and $\mathrm{TXA}_{2}{ }^{8)}$ In addition, repeated antigen inhalation by sensitized rats converts IAR to LAR, a reaction triggered by antigen-specific immunoglobulin $G$ $(\operatorname{IgG}){ }^{9)}$
In the present study, the pharmacological profiles of rat LAR were examined using rat LAR model. To clarify the pharmacological profile of rat LAR, the effects of antiasthmatic drugs including salbutamol ( $\beta_{2}$-agonist), ketotifen (antihistamine), pranlukast (anti-leukotriene $\mathrm{C}_{4} / \mathrm{D}_{4} / \mathrm{E}_{4}$ ), and prednisolone (steroid) were evaluated. In addition, the involvement of cell infiltration in bronchoalveolar lavage fluid (BALF) and pulmonary edema in LAR were investigated in the rat model.

\section{MATERIALS AND METHODS}

Animals Male Sprague-Dawley (SD) rats were purchased from Charles River Japan, Inc. (CRJ, Yokohama, Japan) and individually housed in plastic cages maintained under specific pathogen-free conditions at a room temperature of $23.0 \pm 3.0^{\circ} \mathrm{C}$ and air humidity of $55 \pm 15 \%$ in a 12 -h light/dark cycle environment. The rats were given a standard laboratory chow diet (CRF-1, Oriental Yeast, Tokyo, Japan) and water ad libitum. All experiments complied with the Guidelines for Animal Experimentation of our laboratories.

Compounds The antihistamine ketotifen (Sigma-Aldrich Co.); anti-LT (leukotriene $\mathrm{C}_{4} / \mathrm{D}_{4} / \mathrm{E}_{4}$ receptor antagonist) pranlukast (Ono Pharmaceutical Co.), and steroid prednisolone (Sigma-Aldrich Co.) were prepared in $0.5 \%(\mathrm{w} / \mathrm{v})$ methylcellulose (MC) solution in distilled water, and administered $1 \mathrm{~h}$ before OVA challenge. A vehicle group was administered only $0.5 \% \mathrm{MC}$. A saline group consisted of sensitized rats that were administered $0.5 \% \mathrm{MC}$ and inhaled aerosolized saline.

Salbutamol (Wako, Osaka, Japan) was prepared $0.2 \%$ in saline, and inhaled via nebulizer for $5 \mathrm{~min}$ just before Penh measurement at $3 \mathrm{~h}$ to examine the direct effect on contraction of airway smooth muscle. A vehicle group inhaled saline alone. 
A

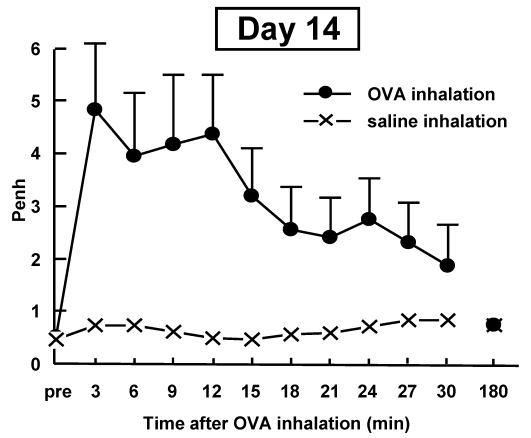

B

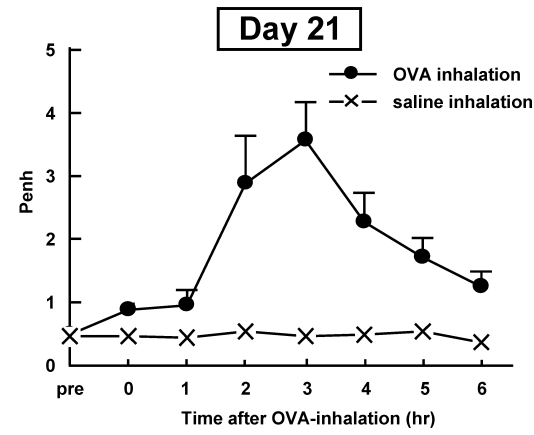

Fig. 1. Changes in Airway Responses after OVA or Saline Inhalation on Day 14 and 21 in Sensitized-Rats

Actively-sensitized rats inhaled $5 \%$ OVA or saline for $5 \mathrm{~min}$ via an ultrasonic nebulizer. After inhalation, Penh was continuously measured by whole-body plethysmography at each time point for $8 \mathrm{~min}$. (A) Time course changes after inhalation on day 14. (B) Time course changes after inhalation on day 21 . Values are the mean \pm S.E. ( $n=6$ ).

OVA-Induced Airway Response SD rats were sensitized on days 0,1 , and 2 by intraperitoneal injection of $10 \mu \mathrm{g}$ of OVA (albumin from chicken egg white grade V, SigmaAldrich Co.) and $10 \mathrm{mg}$ of Alum (Imject Alum, PIERCE) in saline. On day 14 , rats were challenged with exposure to aerosolized $0-5 \%(\mathrm{w} / \mathrm{v})$ OVA in saline for $5 \mathrm{~min}$. Aerosol challenge was performed with groups of up to 4 rats in a closed chamber attached to an ultrasonic nebulizer (NE-U17; Omron Co., Tokyo, Japan). An indicator of airway response (Penh) was measured by barometric plethysmography using whole-body plethysmography (WBP; Buxco, Troy, NY, U.S.A.) under the pre-conditioned room temperature and humidity. ${ }^{10,11)}$ Penh values are a function of the sum of airflows in the airways during the respiratory cycle, ${ }^{12)}$ and potentially existing strain-related differences, such as described in mice. ${ }^{13,14)}$ However, Kirschvink et al. (2005) suggested by comparing Penh with $\mathrm{R}(\mathrm{L})$ that Penh can be used as an index of airflow limitation in spontaneously breathing under welldefined experimental condition in SD rats. ${ }^{15)}$ Rats were placed in the main chamber immediately after challenge, and Penh values were continuously measured for $8 \mathrm{~min}$ and averaged at each time point.

BALF Preparation and Analysis BALFs were collected at each time point after OVA challenge. Briefly, rats were deeply anesthetized by ethyl ether inhalation and exsanguinated from the abdominal aorta. BALF was collected through a bronchial cannula that was connected with a syringe using $8 \mathrm{ml}$ of phosphate-buffered saline (PBS) containing $0.5 \%$ BSA, 2 mM EDTA, and $10 \mu \mathrm{M} \mathrm{AA-861} \mathrm{(5-lipoxyge-}$ nase inhibitor). The number and cell differential of leukocytes in the BALF were determined using a hematology analyzer (ADVIA 120, Bayer Medical, Tokyo, Japan) set in the rat hematology analysis mode.

Measurement of Wet and Dry Lung Weight At various time points after OVA challenge, rats were deeply anesthetized by ethyl ether inhalation and exsanguinated from the abdominal aorta. Whole lung was harvested, and wet weight was measured after trimming of extra tissue. After measuring wet weight, lungs were dried at $60^{\circ} \mathrm{C}$ overnight, and the weight of dried lungs was then measured. Percentage water content in lung was calculated as follows:

Percentage of water contents $=100 \times\{$ (wet lung weight $)-$ (dry lung weight) $\} /$ (wet lung weight).

Statistical Analysis All results are the mean \pm standard

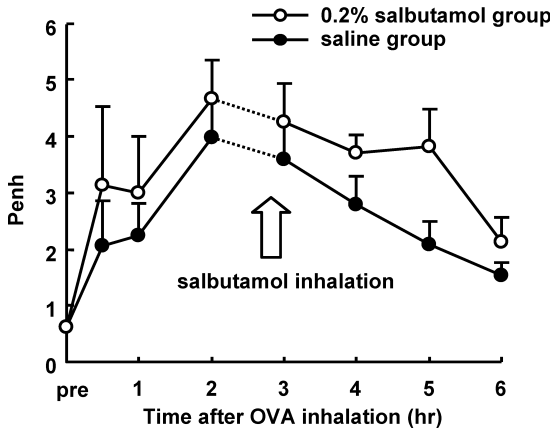

Fig 2. Effects of Inhalation of $0.2 \%(\mathrm{w} / \mathrm{v})$ Salbutamol Solution on Rat LAR After OVA inhalation on day 21, Penh was measured by whole-body plethysmography at each time point for $8 \mathrm{~min}$. Saline or $0.2 \%(\mathrm{w} / \mathrm{v})$ salbutamol solution was inhaled for $5 \mathrm{~min}$ just before measurement of Penh at $3 \mathrm{~h}$ after challenge. Values are the mean \pm S.E. $(n=4$ or 7$)$.

error (S.E.). For comparisons of two groups, the AspinWelch $t$-test (if data were unequal in variance) or Student $t$-test (if data were equal in variance) was used. Findings of $p<0.05$ were considered significant.

\section{RESULTS}

OVA-Induced IAR on Day 14 and LAR on Day 21 The first antigen inhalation on day 14 by sensitized rats increased Penh immediately after challenge, but not at $3 \mathrm{~h}$ after challenge (Fig. 1A). This immediate reaction was nearly completed within $30 \mathrm{~min}$ after challenge. Further, the second antigen inhalation on day 21 gradually increased Penh, which peaked at $3 \mathrm{~h}$ after OVA challenge, while the immediate reaction exhibited on day 14 was decreased in intensity on day 21 (Fig. 1B). Saline inhalation induced no marked responses on either day 14 or day 21 .

BALF Cell Infiltrations in IAR and LAR Comparisons of numbers of BALF cells between IAR and LAR revealed that although numbers of total blood cells and neutrophils did not differ significantly, numbers of eosinophils at 1, 3, and $6 \mathrm{~h}$ in LAR were significantly higher than those of IAR (Table 1).

Pharmacological Profiles of LAR Inhalation of $0.2 \%$ salbutamol at $3 \mathrm{~h}$ after challenge exhibited no effect on Penh (Fig. 2).

The effects of ketotifen, pranlukast, and prednisolone on 
Table 1. Time Course Changes in Numbers of Inflammatory Cells in BALF at IAR or LAR

\begin{tabular}{|c|c|c|c|c|c|c|c|c|}
\hline \multirow{2}{*}{ Cells } & \multicolumn{2}{|l|}{ Group } & \multicolumn{6}{|c|}{ Time after OVA inhalation } \\
\hline & Day & Inhala & Pre & $0 \mathrm{~min}$ & $1 \mathrm{~h}$ & $3 \mathrm{~h}$ & $6 \mathrm{~h}$ & $24 \mathrm{~h}$ \\
\hline \multirow{4}{*}{$\begin{array}{l}\text { Total blood cells } \\
\left(\times 10^{6} \text { cells/lung) }\right.\end{array}$} & 14 & Saline & $1.0 \pm 0.2$ & $1.2 \pm 0.3$ & $0.8 \pm 0.1$ & $0.9 \pm 0.2$ & $0.8 \pm 0.1$ & $1.1 \pm 0.1$ \\
\hline & (IAR) & OVA & & $0.8 \pm 0.1$ & $0.7 \pm 0.1$ & $1.4 \pm 0.1$ & $2.0 \pm 0.4$ & $1.3 \pm 0.3$ \\
\hline & & Saline & $1.2 \pm 0.3$ & $0.9 \pm 0.2$ & $1.0 \pm 0.1$ & $0.9 \pm 0.2$ & $0.9 \pm 0.0$ & $0.7 \pm 0.1$ \\
\hline & (LAR) & OVA & & $1.0 \pm 0.1$ & $1.1 \pm 0.2$ & $1.6 \pm 0.4$ & $3.4 \pm 0.9$ & $4.8 \pm 2.1$ \\
\hline \multirow{4}{*}{$\begin{array}{l}\text { Neutrophils } \\
\left(\times 10^{6} \text { cells/lung) }\right.\end{array}$} & 14 & Saline & $0.2 \pm 0.0$ & $0.3 \pm 0.1$ & $0.3 \pm 0.0$ & $0.3 \pm 0.1$ & $0.4 \pm 0.0$ & $0.4 \pm 0.1$ \\
\hline & (IAR) & OVA & & $0.2 \pm 0.0$ & $0.2 \pm 0.0$ & $0.7 \pm 0.2$ & $1.1 \pm 0.3$ & $1.2 \pm 0.6$ \\
\hline & & Saline & $0.3 \pm 0.1$ & $0.2 \pm 0.1$ & $0.3 \pm 0.1$ & $0.2 \pm 0.0$ & $0.3 \pm 0.0$ & $0.3 \pm 0.0$ \\
\hline & (LAR) & OVA & & $0.2 \pm 0.0$ & $0.2 \pm 0.1$ & $0.6 \pm 0.2$ & $2.1 \pm 0.9$ & $2.3 \pm 1.1$ \\
\hline \multirow{4}{*}{$\begin{array}{l}\text { Eosinophils } \\
\left(\times 10^{5} \text { cells/lung }\right)\end{array}$} & 14 & Saline & $0.4 \pm 0.2$ & $0.0 \pm 0.0$ & $0.0 \pm 0.0$ & $0.0 \pm 0.0$ & $0.4 \pm 0.2$ & $0.0 \pm 0.0$ \\
\hline & (IAR) & OVA & & $0.0 \pm 0.0$ & $0.0 \pm 0.0$ & $0.0 \pm 0.0$ & $0.1 \pm 0.1$ & $2.0 \pm 1.0$ \\
\hline & & Saline & $1.1 \pm 0.8$ & $0.0 \pm 0.0$ & $0.3 \pm 0.2$ & $0.2 \pm 0.2$ & $0.3 \pm 0.2$ & $0.0 \pm 0.0$ \\
\hline & (LAR) & OVA & & $0.5 \pm 0.2$ & $0.6 \pm 0.2^{\#}$ & $1.0 \pm 0.3^{\#}$ & $1.9 \pm 0.7^{\#}$ & $2.8 \pm 1.2$ \\
\hline
\end{tabular}

Values are the mean \pm S.E. ( $n=4$ to 5 ). ${ }^{\#} p<0.05$ ( $v s$. respective IAR by Student $t$-test).

A

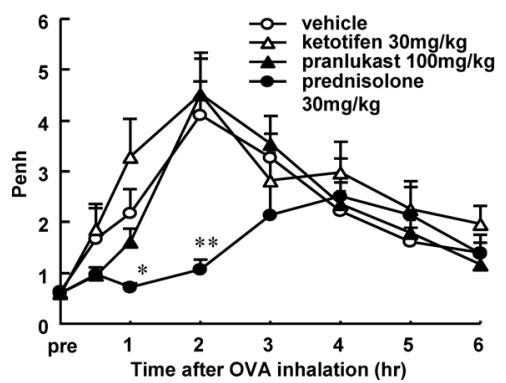

B

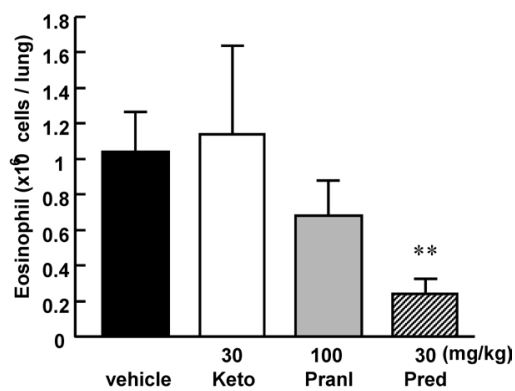

Fig. 3. The Effects of Ketotifen, Pranlukast, and Prednisolone on Rat LAR and Numbers of Eosinophils in BALF

One hour before challenge, rats were orally administered ketotifen $30 \mathrm{mg} / \mathrm{kg}$, pranlukast $30 \mathrm{mg} / \mathrm{kg}$, or prednisolone $30 \mathrm{mg} / \mathrm{kg}$. Penh was measured by whole-body plethysmography at each time point for $8 \mathrm{~min}$. Twenty-four hours after OVA inhalation, BALF was collected, and numbers of eosinophils in BALF were determined using a hematology analyzer. (A) Time course changes after OVA inhalation; (B) numbers of eosinophils in BALF at $24 \mathrm{~h}$ after challenge. Keto: ketotifen, Pranl: pranlukast, Pred: prednisolone. Values are the mean \pm S.E. $(n=6-15)$. $* * * p<0.05,0.01$ (significantly different from vehicle group by Aspin-Welch $t$-test)

antigen-induced airway responses are shown in Fig. 3A. Oral administration of ketotifen $30 \mathrm{mg} / \mathrm{kg}$ or pranlukast $100 \mathrm{mg} / \mathrm{kg}$ had no effect on LAR, while prednisolone $30 \mathrm{mg} / \mathrm{kg}$ significantly attenuated increase in Penh.

The effects of drugs on antigen-induced eosinophil infiltrations in BALF are shown in Fig. 3B. Ketotifen and pranlukast exhibited no significant effects on eosinophil infiltration. However, prednisolone significantly decreased the number of eosinophils in BALF. Although prednisolone tended to decrease total numbers of blood cells and numbers of neutrophils in BALF, these effects were not significant (data not shown).

Time Course Changes in Lung Water Content In the OVA inhalation group, which exhibited LAR, the percentage of water content in lung was significantly increased at $3 \mathrm{~h}$ after OVA challenge compared to that in the saline inhalation group (Fig. 4).

\section{DISCUSSION}

In the present study, the pharmacological and pathophysiological characteristics of rat antigen-induced LAR were examined. Only the numbers of eosinophils during LAR were significantly higher than those during IAR. Prednisolone at-

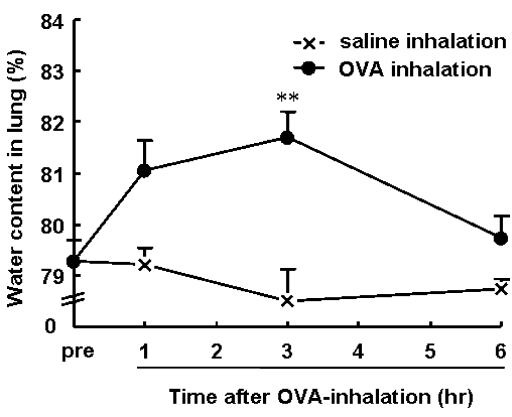

Fig. 4. Time Course Changes in the Percentage of Water Content in Lung after Saline or OVA Inhalation on Day 21

At various time point after challenge, rats were deeply anesthetized and exsanguinated from the abdominal aorta. Whole lung was harvested, and wet weight was measured after trimming of extra tissue. After measuring wet weight, lungs were dried at $60^{\circ} \mathrm{C}$ overnight, and the weight of dried lungs was then measured. Percentages of water content in lung were calculated from wet lung weight and dry lung weight. Values are the mean \pm S.E.M. $(n=3-6)$. $* * p<0.01$ (significantly different from saline inhalation group)

tenuated LAR and eosinophil infiltration, while salbutamol, ketotifen, and pranlukast each exhibited no effect on LAR. Further, the percentage of water contents in lung increased $3 \mathrm{~h}$ after challenge. These findings suggest that antigen-induced LAR is a response involving pulmonary edema and 
not contraction of airway smooth muscle. This pulmonary edema is not mediated by histamine or leukotriene $\mathrm{C}_{4} / \mathrm{D}_{4} / \mathrm{E}_{4}$, but might involve eosinophil infiltration.

The involvement of cell infiltration in antigen-induced LAR has thus far been examined using animal models. ${ }^{16,17)}$ However, most animal models including those in mouse and guinea pig exhibit both IAR and LAR with the same antigen inhalation, making it difficult to directly examine the relationships between cell infiltration and airway response. In our rat model, since IAR and LAR were independently induced on different days, differences between IAR and LAR could be clearly determined. During LAR, eosinophil number but not neutrophil number was significantly higher than during IAR. These findings suggest that infiltration of eosinophils into lung is involved in the induction of antigen-induced LAR but not IAR.

It was reported that metyrapone, an $11 \beta$-hydroxylase inhibitor, decreased OVA-induced rat $\mathrm{LAR}^{18)}$ and that pranlukast inhibited $\mathrm{CD}^{+}{ }^{+} \mathrm{T}$ cell-driven rat $\mathrm{LAR},{ }^{19)}$ while OVA plus zymosan-activated serum induced rat LAR. ${ }^{20)}$ Further, pretreatment with montelukast or methylprednisolone inhibited OVA-induced LAR under anesthesia by reducing the production of leukotrienes in the lung. ${ }^{21)}$ However, the effects of $\beta_{2}$-agonist and antihistamine on rat LAR have not been previously evaluated. In the present study, salbutamol, ketotifen, and pranlukast exhibited no effects on either LAR or eosinophil numbers in BALF, whereas prednisolone significantly suppressed both LAR and eosinophil number (Figs. 2, 3) The doses of salbutamol, ketotifen and pranlukast used in this study were considered sufficient for examination, since they completely suppress antigen-induced immediate reactions in rats. ${ }^{22}$ In our previous study, salbutamol completely suppressed the rat IAR, whereas ketotifen and pranlukast partially inhibited. ${ }^{8)}$ These findings thus indicated that bronchoconstriction play a role in rat IAR but not in rat LAR, consistent with the findings of a previous study in guinea pig. ${ }^{4,6}$ On the other hand, LT is involved in rat IAR but not in rat LAR. In clinical studies of asthma, anti-LTs reduced antigen-induced LAR by only about $50 \%,{ }^{23,24)}$ suggesting that other mechanisms not related to LT are involved in LAR. Further studies are needed to examine the mediators involved in LAR.

Percentage water content in lung was significantly increased at $3 \mathrm{~h}$ (Fig. 4), indicating that pulmonary edema plays a role in LAR. Submucosal edema was observed in Ascaris suum extract-induced guinea pig LAR, ${ }^{25)}$ and repeated exposure to OVA in sensitized guinea pigs induced perivascular edema. ${ }^{26)}$ Moreover, increase in vascular permeability may be an important factor in the pathogenesis of Ascaris suum antigen-induced dog LAR. ${ }^{27)}$ These previous results are consistent with our finding that pulmonary edema is observed in antigen-induced LAR.

Activated eosinophils are potent effector cells and can cause pulmonary vasoconstriction, bronchoconstriction, and vascular endothelial injury without widespread plugging of capillaries by aggregated eosinophils. ${ }^{28)}$ It has also been reported that eosinophil peroxidase (EPO) altered airway epithelial permeability in the guinea pig. ${ }^{29)}$ The role of eosinophils in the clinical manifestation of asthma has recently been questioned, because anti-interleukin (IL)-5 therapy couldn't improve clinical symptoms in patients with asthma. In these clinical study, eosinophils in peripheral blood were largely depleted after anti-IL-5 treatment, however residual eosinophilia in tissue persisted, which permits speculation that the remaining eosinophils may be sufficient to drive the asthma symptomatology. ${ }^{30)}$ In the case of rat LAR, numbers of eosinophils in the induction phase, such as $1,3,6 \mathrm{~h}$ after challenge, were significantly higher than those of IAR, but numbers at $24 \mathrm{~h}$ weren't. We have reported that these observations suggest that the eosinophils in lung might have some role in the early induction phase of antigen-induced LAR.

In conclusion, antigen-induced LAR is a response involving pulmonary edema and not contraction of airway smooth muscle. Moreover, this pulmonary edema is not mediated by histamine or leukotriene $\mathrm{C}_{4} / \mathrm{D}_{4} / \mathrm{E}_{4}$, but might involve eosinophil infiltration. This is the first comprehensive study of the profiles of rat antigen-induced LAR, and these analyses of rat LAR improve understanding of the diverse mechanisms underlying human asthmatic diseases.

\section{REFERENCES}

1) Barnes P. J., Chung K. F., Page C. P., Pharmacol. Rev., 50, 515 (1998).

2) Pepys J., Hutchcroft B. J., Am. Rev. Respir. Dis., 112, 829 (1975).

3) Nagy L., Lee T. H., Kay A. B., N. Engl. J. Med., 306, 497-501 (1982).

4) Justesen D. R., Braun E. W., Garrison R. G., Pendleton R. B., Science, 170, 864-866 (1970).

5) Nakagawa N., Obata T., Kobayashi T., Okada Y., Nambu F., Terawaki T., Furuya T., Muryobayashi K., Sawada M., Aishita H., Eur. J. Pharmacol., 235, 211-219 (1993).

6) Matsumoto T., Ashida Y., Tsukuda R., J. Pharmacol. Exp. Ther, 269, 1236 - 1244 (1994)

7) Akutsu I., Kojima T., Kariyone A., Fukuda T., Makino S., Takatsu K., Immunol. Lett., 45, 109-16 (1995).

8) Miyagawa N., Iwasaki H., Kato T., Tanaka M., Shibata T., Wakitani K., Biol. Pharm. Bull., 31, 2260-2264 (2008).

9) Miyagawa N., Iwasaki H., Kato T., Tanaka M., Shibata T., Wakitani K., Intern. Immunopharmacol., 8, 1848-1853 (2008).

10) Hamelmann E., Schwarze J., Takeda K., Oshiba A., Larsen G. L., Irvin C. G., Gelfand E. W., Am. J. Respir. Crit. Care Med., 156, 766-775 (1997).

11) Elekes K., Helyes Z., Kereskai L., Sándor K., Pintér E., Pozsgai G., Tékus V., Bánvölgyi A., Németh J., Szuts T., Kéri G., Szolcsányi J., Eur. J. Pharmacol., 578, 313-322 (2008).

12) Zhang X. D., Andrew M. E., Hubbs A. F., Siegel P. D., Toxicol. Sci. 94, 322-329 (2006).

13) Adler A., Cieslewicz G., Irvin C. G., J. Appl. Physiol., 97, 286-292 (2004).

14) Duguet A., Biyah K., Minshall E., Gomes R., Wang C. G., TaoudiBenchekroun M., Bates J. H., Eidelman D. H., Am. J. Respir. Crit Care Med., 161, 839-848 (2000).

15) Kirschvink N., Vincke G., Onclinx C., Peck M. J., Gustin P., J. Pharmacol. Toxicol. Methods, 51, 123-128 (2005).

16) Uyama O., Ihaku D., Kitada O., Miyasaka M., Sugita M., Res. Commun. Mol. Pathol. Pharmacol., 90, 3-15 (1995).

17) Zhang X. D., Andrew M. E., Hubbs A. F., Siegel P. D., Toxicol. Sci., 32, 322-329, Epub 2006 Sep 18 (2006).

18) Turner D. J., Myron P., Powell W. S., Martin J. G., Am. J. Respir. Crit Care Med., 153, 545-50 (1996)

19) Hojo M., Suzuki M., Maghni K., Hamid Q., Powell W. S., Martin J. G., J. Pharmacol. Exp. Ther., 293, 410-416 (2000).

20) Kodani M., Sakata N., Takano Y., Kamiya H., Katsuragi T., Hugli T. E., Abe M., Immunopharmacology, 49, 263-274 (2000)

21) Murai A., Abe M., Hayashi Y., Sakata N., Katsuragi T., Tanaka K., J. Pharmacol. Exp. Ther, 312, 432-440, Epub 2004 Oct 6 (2005).

22) Ueno M., Inagaki N., Nagai H., Koda A., Pharmacology, 57, 206 214 (1998).

23) Roquet A., Dahlen B., Kumlin M., Ihre E., Anstren G., Binks S., Dahlen S. E., Am. J. Respir. Crit. Care Med., 155, 1856-1863 (1997).

24) Obase Y., Shimoda T., Matsuo N., Matsuse H., Asai S., Kohno S. 
Chest, 114, 1028-1032 (1998).

25) Iijima H., Ishii M., Yamauchi K., Chao C. L., Kimura K., Shimura S., Shindoh Y., Inoue H., Mue S., Takishima T., Am. Rev. Respir. Dis., 136, 922-929 (1987).

26) Takizawa T., Watanabe C., Saiki I., Wada Y., Tohma T., Nagai H., Biol. Pharm. Bull., 24, 1127-1132 (2001).

27) Ohrui T., Sekizawa K., Aikawa T., Yamauchi K., Sasaki H., Takishima
T., J. Allergy Clin. Immunol., 89, 933-943 (1992).

28) Fujimoto K., Parker J. C., Kayes S. G., Am. Rev. Respir. Dis., 142, 1414-1421 (1990).

29) Brottman GM., Regelmann W. E., Slungaard A., Wangensteen O. D., Pediatr. Pulmonol., 21, 159-166 (1996).

30) Rådinger M., Lötvall J., Pharmacol. Ther., in press (2008). 